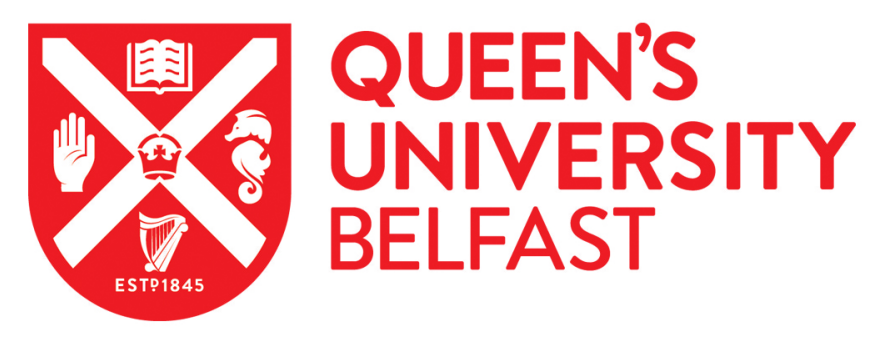

\title{
Dynamics and structure of self-generated magnetics fields on solids following high contrast, high intensity laser irradiation
}

Albertazzi, B., Chen, S. N., Antici, P., Böker, J., Borghesi, M., Breil, J., Dervieux, V., Feugeas, J. L., Lancia, L., Nakatsutsumi, M., Nicolaï, P., Romagnagni, L., Shepherd, R., Sentoku, Y., Starodubtsev, M., Swantusch, M., Tikhonchuk, V. T., Willi, O., D'Humières, E., ... Fuchs, J. (2015). Dynamics and structure of self-generated magnetics fields on solids following high contrast, high intensity laser irradiation. Physics of Plasmas, 22(12), [123108]. https://doi.org/10.1063/1.4936095

Published in:

Physics of Plasmas

Document Version:

Publisher's PDF, also known as Version of record

Queen's University Belfast - Research Portal:

Link to publication record in Queen's University Belfast Research Portal

Publisher rights

Copyright (2015) AIP Publishing. This article may be downloaded for personal use only. Any other use requires prior permission of the author and AIP Publishing.

The following article appeared in Albertazzi, B, Chen, SN, Antici, P, Böker, J, Borghesi, M, Breil, J, Dervieux, V, Feugeas, JL, Lancia, L, Nakatsutsumi, M, Nicolaï, P, Romagnagni, L, Shepherd, R, Sentoku, Y, Starodubtsev, M, Swantusch, M, Tikhonchuk, VT, Willi, O,

D'Humières, E, Pépin, H \& Fuchs, J 2015, 'Dynamics and structure of self-generated magnetics fields on solids following high contrast, high intensity laser irradiation' Physics of Plasmas, vol 22, no. 12, 123108 and may be found at

http://scitation.aip.org/content/aip/journal/pop/22/12/10.1063/1.4936095

\section{General rights}

Copyright for the publications made accessible via the Queen's University Belfast Research Portal is retained by the author(s) and / or other copyright owners and it is a condition of accessing these publications that users recognise and abide by the legal requirements associated with these rights.

Take down policy

The Research Portal is Queen's institutional repository that provides access to Queen's research output. Every effort has been made to ensure that content in the Research Portal does not infringe any person's rights, or applicable UK laws. If you discover content in the Research Portal that you believe breaches copyright or violates any law, please contact openaccess@qub.ac.uk. 


\section{AIP $\left.\right|_{\text {Physics of }}$

Dynamics and structure of self-generated magnetics fields on solids following high contrast, high intensity laser irradiation

B. Albertazzi, S. N. Chen, P. Antici, J. Böker, M. Borghesi, J. Breil, V. Dervieux, J. L. Feugeas, L. Lancia, M. Nakatsutsumi, Ph. Nicolaï, L. Romagnagni, R. Shepherd, Y. Sentoku, M. Starodubtsev, M. Swantusch, V. T. Tikhonchuk, O. Willi, E. d'Humières, H. Pépin, and J. Fuchs

Citation: Physics of Plasmas 22, 123108 (2015); doi: 10.1063/1.4936095

View online: http://dx.doi.org/10.1063/1.4936095

View Table of Contents: http://scitation.aip.org/content/aip/journal/pop/22/12?ver=pdfcov

Published by the AIP Publishing

\section{Articles you may be interested in}

Self-generated magnetic fields in q-distributed plasmas

Phys. Plasmas 20, 022308 (2013); 10.1063/1.4793455

Dynamics of thin metal foils irradiated by moderate-contrast high-intensity laser beams

Phys. Plasmas 19, 023110 (2012); 10.1063/1.3683687

Electromagnetic pulse reflection at self-generated plasma mirrors: Laser pulse shaping and high order harmonic generation

Phys. Plasmas 14, 093105 (2007); 10.1063/1.2776906

Effects of self-generated magnetic fields and nonlocal electron transport in laser produced plasmas Phys. Plasmas 7, 4250 (2000); 10.1063/1.1289895

Self-generated magnetic field by transverse plasmons in laser-produced plasma

Phys. Plasmas 7, 3405 (2000); 10.1063/1.874204

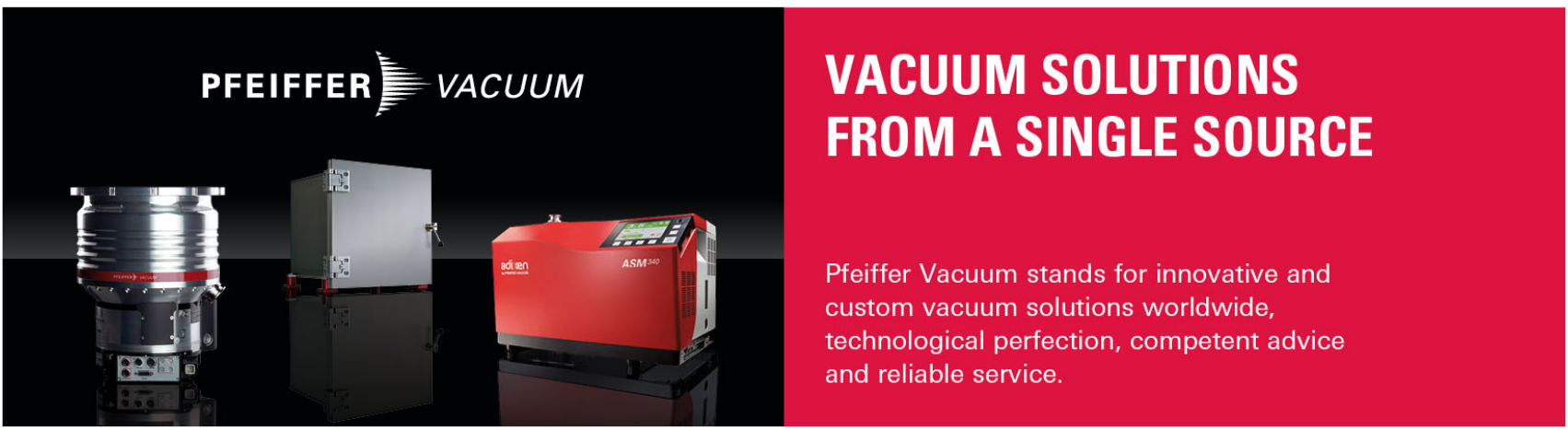




\title{
Dynamics and structure of self-generated magnetics fields on solids following high contrast, high intensity laser irradiation
}

\author{
B. Albertazzi, ${ }^{1,2,3}$ S. N. Chen, ${ }^{1,4}$ P. Antici, ${ }^{2,5}$ J. Böker, ${ }^{6}$ M. Borghesi, ${ }^{7}$ J. Breil, ${ }^{8}$ V. Dervieux, ${ }^{1}$ \\ J. L. Feugeas, ${ }^{8}$ L. Lancia, ${ }^{5}$ M. Nakatsutsumi, ${ }^{1}$ Ph. Nicolaï, ${ }^{8}$ L. Romagnagni, ${ }^{1}{ }^{1}$ R. Shepherd, ${ }^{9}$ \\ Y. Sentoku, ${ }^{10}$ M. Starodubtsev,${ }^{4}$ M. Swantusch, ${ }^{6}$ V. T. Tikhonchuk, ${ }^{8}$ O. Willi, ${ }^{6}$ \\ E. d'Humières, ${ }^{8}$ H. Pépin, ${ }^{2}$ and J. Fuchs ${ }^{1,4, a)}$ \\ ${ }^{1}$ LULI, École Polytechnique, CNRS, CEA, UPMC, 91128 Palaiseau, France \\ ${ }^{2}$ INRS-EMT, 1650 bd L. Boulet, J3X1S2, Varennes, Québec, Canada \\ ${ }^{3}$ Graduate School of Engineering, University of Osaka, Suita, Osaka 565-087, Japan \\ ${ }^{4}$ Institute of Applied Physics, 46 Ulyanov Street, 603950 Nizhny Novgorod, Russia \\ ${ }^{5}$ Dept. SBAI, Universita di Roma “La Sapienza," Via A. Scarpa 14, 00161 Rome, Italy \\ ${ }^{6}$ Institut für Laser-und Plasmaphysik, Heinrich-Heine-Universität, Düsseldorf, Germany \\ ${ }^{7}$ School of Mathematics and Physics, The Queen's University, Belfast, United Kingdom \\ ${ }^{8}$ CELIA, University of Bordeaux - CNRS - CEA, 33405 Talence, France \\ ${ }^{9}$ LLNL, East Av., Livermore, California 94550, USA \\ ${ }^{10}$ Department of Physics, University of Nevada, Reno, Nevada 89557-0058, USA
}

(Received 7 July 2015; accepted 11 October 2015; published online 9 December 2015)

The dynamics of self-generated magnetic B-fields produced following the interaction of a high contrast, high intensity $\left(\mathrm{I}>10^{19} \mathrm{~W} \mathrm{~cm}^{-2}\right)$ laser beam with thin $(3 \mu \mathrm{m}$ thick) solid ( $\mathrm{Al}$ or $\mathrm{Au})$ targets is investigated experimentally and numerically. Two main sources drive the growth of B-fields on the target surfaces. B-fields are first driven by laser-generated hot electron currents that relax over 10-20 ps. Over longer timescales, the hydrodynamic expansion of the bulk of the target into vacuum also generates B-field induced by non-collinear gradients of density and temperature. The laser irradiation of the target front side strongly localizes the energy deposition at the target front, in contrast to the target rear side, which is heated by fast electrons over a much larger area. This induces an asymmetry in the hydrodynamic expansion between the front and rear target surfaces, and consequently the associated B-fields are found strongly asymmetric. The sole long-lasting ( $>30$ ps) B-fields are the ones growing on the target front surface, where they remain of extremely high strength $(\sim 8-10 \mathrm{MG})$. These B-fields have been recently put by us in practical use for focusing laser-accelerated protons [B. Albertazzi et al., Rev. Sci. Instrum. 86, 043502 (2015)]; here we analyze in detail their dynamics and structure. (C) 2015 AIP Publishing LLC.

[http://dx.doi.org/10.1063/1.4936095]

\section{INTRODUCTION}

Self-generated magnetic fields (B-fields), following the interaction between an ultra-short, ultra-high intensity (i.e., having typical duration $\leq 1 \mathrm{ps}$ and intensity $\geq 10^{18} \mathrm{~W} \mathrm{~cm}^{-2}$ $\mu \mathrm{m}^{2}$ ) laser beam and a solid target, can be of extremely large strength, i.e., in the several MegaGauss (MG) range. They have been the subject of numerous investigations, both experimental and theoretical, which were mostly motivated by the strong influence these fields can have on the evolution of laser-produced plasmas and laser-produced fast electrons. ${ }^{1-10}$ These B-fields can be used for applications, e.g., to focus laser-generated energetic and high-current charged particle beams. In particular, we have recently investigated ${ }^{11}$ exploiting such B-fields to produce a high-density focused proton beam from an initially divergent source ${ }^{12}$ generated through the Target Normal Sheath Acceleration (TNSA ${ }^{13}$ ) mechanism from a laser-irradiated solid target.

These B-fields have been initially experimentally investigated by measuring polarization rotation of light either emitted from the plasma ${ }^{14}$ or probing it. ${ }^{15,16}$ More recently,

\footnotetext{
a)julien.fuchs@polytechnique.fr
}

Schumaker et al. ${ }^{17}$ probed such B-fields using a high-energy, wakefield-accelerated electron beam, by taking advantage of the deflections induced on the probing particles by the fields they cross. The obvious advantage of using high-energy charged particles compared with optical probe is that the former can probe the B-fields through solid targets, i.e., even B-fields developing within the solid matter. Since such selfgenerated B-fields have a toroidal structure, ${ }^{1-11}$ with the axis of the torus being aligned with the target normal, the most effective way to probe the fields is to send the particles along the torus axis, i.e., "face-on," so that they can be deflected by the Lorentz force $q(\mathbf{v} \times \mathbf{B})$. This way, the probing particles will also be less sensitive to E-fields, which are generally oriented along the target normal. ${ }^{18}$ Alternatively to using electrons, protons, either monochromatic ${ }^{19}$ as produced by nuclear reactions or broadband ${ }^{20}$ as produced by the TNSA mechanism, can be used to radiograph B-fields. Of particular interest are the latter since, being broadband and nonrelativistic, the probing protons quickly spread in time from the source to the object to be probed. Hence, by resolving them in energy after they have crossed the B-field structure, one can record, in a single shot, the evolution of the fields 
over a time span that corresponds to the overall proton beam temporal spread up to the B-field structure. ${ }^{20,21}$

The goal of the present paper is to analyze in detail, using the proton radiography technique coupled to numerical simulations, the dynamics and structure of the B-fields we used in the proton focusing device ${ }^{11}$ mentioned earlier. Compared with our previous investigation, ${ }^{21}$ we use here a laser having high-temporal contrast to trigger the B-field growth. This prevents the formation of a pre-plasma at the target front side, and thus allows us to observe and separate the various mechanisms inducing B-field generation, i.e., field generation by hot electron currents and by target hydrodynamic expansion. This is possible since the B-field probing is also performed over longer times than in Ref. 21. We will also show that over tens of ps only a strong B-field remains on the front target surface. This is essentially due to the strong asymmetry between the target front and rear sides induced by the laser irradiation at the target front: as there the laser energy has been deposited over a small area (of the order of the laser focal spot, i.e., a few $\mu \mathrm{m}^{2}$ ), the temperature gradients are strong and so are the induced B-fields (of the order of 10 s of MG). In contrast, the target rear side is heated over a much larger area $\left(\sim 10^{4} \mu \mathrm{m}^{2}\right)^{22}$ by fast electrons that are widely divergent from the target front. Hence, the temperature gradients are there smoother, resulting in weaker surface B-fields (of the order of 10 s of $\mathrm{kG}$ ).

This paper is organized as follows. In Section II, we present the results of the experiment conducted to measure the B-fields while Section III is devoted to the analysis of the topology and evolution of the B-fields inferred from the experimental data. In Section III A, we discuss the various mechanisms through which B-fields can be self-generated following the high-intensity laser-target interaction. Section III B focuses on the numerical simulations performed using the Particle-In-Cell code PICLS and on the comparisons with the experimental data of the B-fields induced at early time by the hot electron currents within the target. In Section III C, we investigate the transition between the B-fields induced by hot electrons and the thermoelectric B-fields, i.e., the ones induced by the hydrodynamic expansion of the plasma. $^{23}$ Section IIID presents further discussion on the analytical and numerical analysis of the thermoelectric B-fields, and the comparison with the experimental results. Section IV concludes the study.

\section{EXPERIMENTAL RESULTS}

We here briefly recall the setup and main results of the experiment which were already presented in Ref. 11 as we will use them to conduct the discussion on the B-field structure and dynamics.

\section{A. Experimental setup}

The experiment was performed using the Titan laser at the Jupiter Laser Facility located at the Lawrence Livermore National Laboratory (USA). The short-pulse arm of Titan was split in two inside the target chamber to produce two high intensity beams. As shown in Figure 1, the first laser beam (B1, having an intensity $\mathrm{I}>10^{19} \mathrm{~W} \mathrm{~cm}^{-2}$ ) irradiated

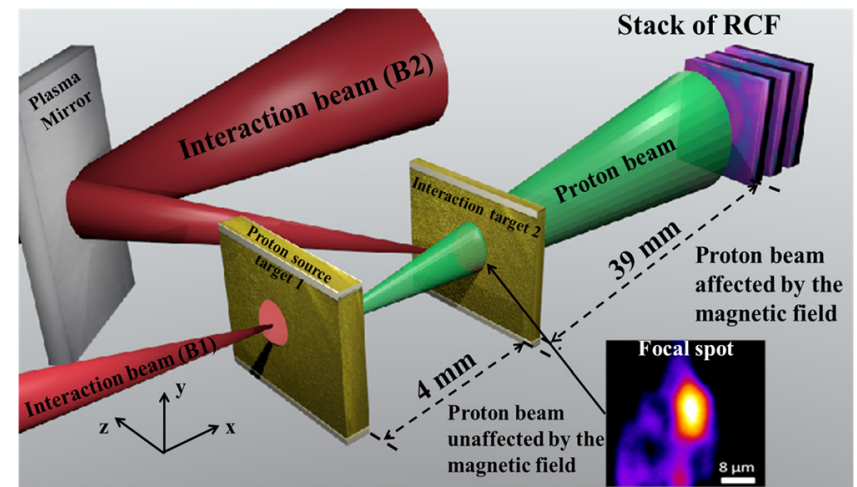

FIG. 1. Experimental set-up (see text). In the experiment, target \#2 was varied in thickness and in material ( $\mathrm{Au}$ or $\mathrm{Al}$ ).

target \#1 (Au $50 \mu \mathrm{m}$ thick) from which, through the TNSA mechanism, a proton beam having maximum energy of $15 \mathrm{MeV}$ was accelerated.

The second laser beam (B2) was used to trigger the selfgeneration of the B-fields on target \#2. B2 was incident on target $\# 2$ at an angle of $\sim 30^{\circ}$ from the target normal. Each laser beam had an energy of approximately $55 \mathrm{~J} \pm 10 \%$, a pulse duration of $\sim 700$ fs full width at half maximum (FWHM) and was focused down to $8-10 \mu \mathrm{m}$. Prior to its focusing on target \#2, B2 was reflected off a plasma mirror (PM) ${ }^{24}$ to improve its temporal contrast, i.e., to remove the long-duration energy pedestal prior to the $700 \mathrm{fs}$ duration main pulse. This prevented the formation of plasma, induced by the pedestal, at the front of target \#2. Such plasma, expanding into vacuum, would have induced the growth of thermoelectric B-fields prior to the irradiation by the peak of the laser pulse, thus preventing us from probing the B-fields triggered solely by the action of the highintensity irradiation of target \#2 by B2. The plasma mirror has an efficiency of $70 \%$, which resulted in B2 having an on-target intensity of $\sim 2-3 \times 10^{19} \mathrm{~W} \mathrm{~cm}^{-2}$.

As shown in Figure 1, the proton beam accelerated from target \#1 was used to probe the spatial distribution and strength of the B-fields on target \#2. The two targets were parallel to each other to allow "face-on" probing. The proton deflections are projected onto a stack of radiochromic films $(\mathrm{RCFs}),{ }^{12}$ with a projection factor that is equal to $(\mathrm{d}+\mathrm{D}) / \mathrm{d}$ where $d=4 \mathrm{~mm}$ was the distance from target \#1 to target \#2, and $\mathrm{D}=39 \mathrm{~mm}$ is the distance from target \#2 to the RCF. In our case, the projection factor is 10.75 .

Each RCF film detects a narrow energy range of the incident protons $(\mathrm{E} \simeq 0.5 \mathrm{MeV})$ due to the Bragg peak associated with the energy deposition of protons in matter. ${ }^{25}$ As a result of the difference in time of flight of protons within this $0.5 \mathrm{MeV}$ energy range, the temporal integration through target \#2 of the probing protons in each film is $\sim 2$ ps at $9.5 \mathrm{MeV}$, i.e., for early probing times, and $\sim 15$ ps at 4.5 MeV, i.e., for late probing times, i.e., shorter than the B-fields evolution timescale in each phase of their evolution, which we will detail later.

There are also protons accelerated from target \#2 and incident on the RCF, but these, at the energies we analyze, are of negligible dose compared with the ones produced from target \#1, as shown by comparing Figures 2(q) and 2(r). 


\section{B. Results}

The experimental proton deflection maps obtained with a $3 \mu \mathrm{m}$ thick $\mathrm{Al}$ foil and a $3 \mu \mathrm{m}$ thick Au foil as target $\# 2^{11}$ are shown, respectively, in Figures 3(a) and 3(i). Target \#2 is kept at a minimum thickness, otherwise multiple scattering ${ }^{26}$ of the probing protons induced by passing through the solid would blur the field-induced deflections. At all times, the proton deflection structure is circular, indicating an axi-symmetric B-field structure (with its symmetry axis along the target normal), which is consistent with the toroidal topology mentioned earlier and with previous experimental results and theory. ${ }^{1-11}$
At $\mathrm{t} \sim 0$ (i.e., the time at which B2 is irradiating target \#2), we observe for both types of target a similar depletion of protons in the center of the deflection structure, with the protons being accumulated in an outer ring in a similar way. This characteristic pattern disappears quickly in the case of the $\mathrm{Al}$ target, but persists up to $t=8 \mathrm{ps}$ for the Au target. At later times $(\mathrm{t} \geq 4$ ps for the $\mathrm{Al}$ target and $\mathrm{t} \geq 14$ ps for the Au target), a strong accumulation of protons appears in the center of the structure. Simultaneously, the outer proton accumulation ring progressively vanishes. In the case of the $\mathrm{Al}$ target, after $13.5 \mathrm{ps}$, the outer ring disappears completely and only the central proton accumulation spot remains up to the last probing time ( $\sim 43 \mathrm{ps})$.
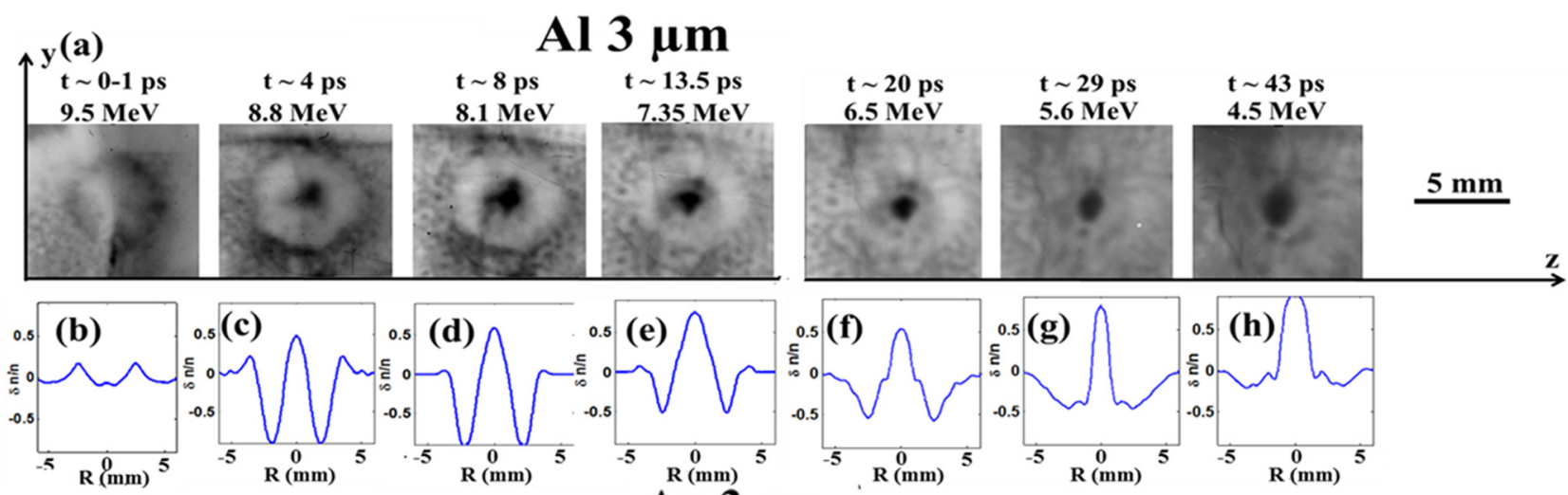

(i)
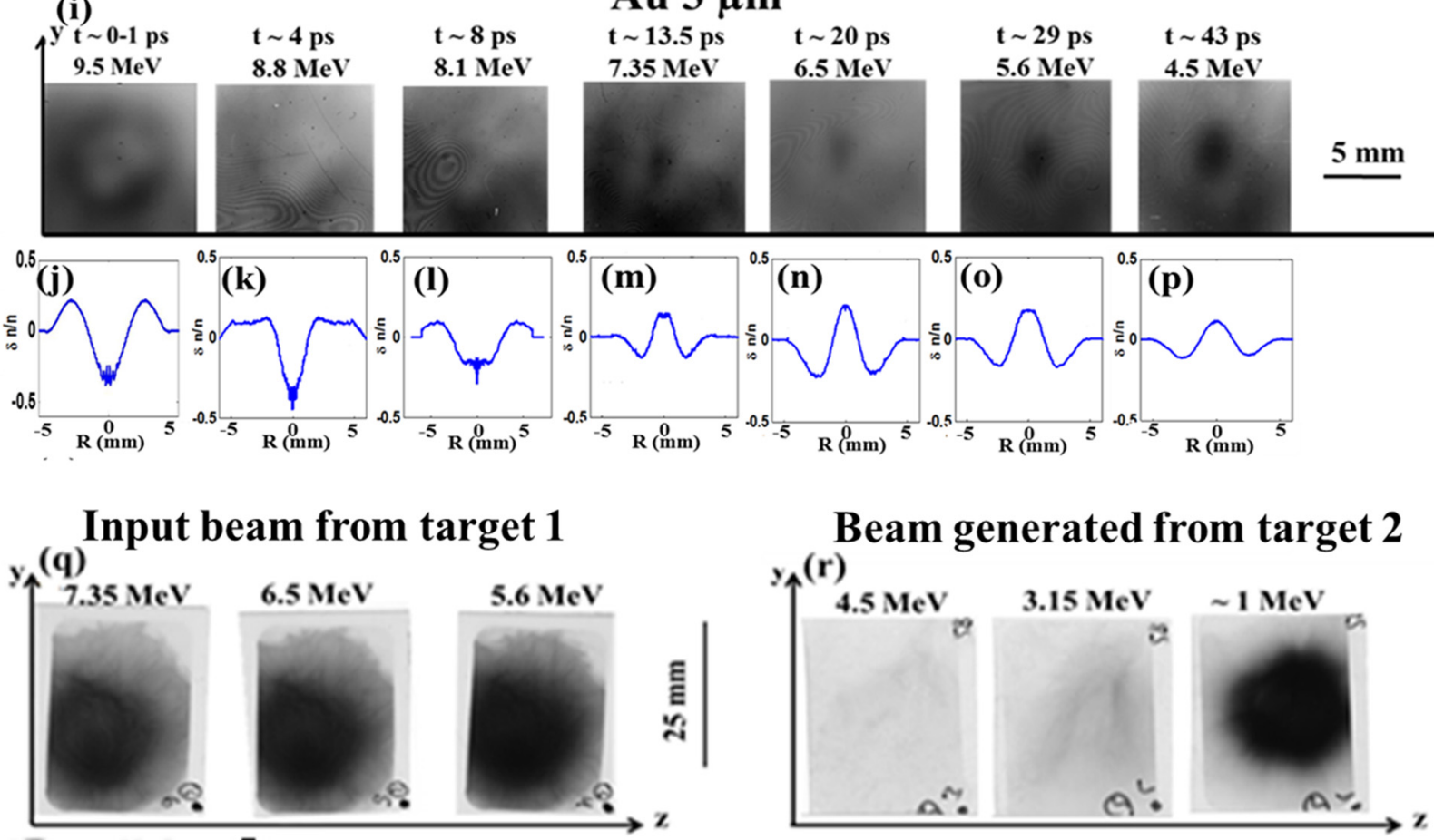

FIG. 2. (a) Proton deflection maps as recorded on RCF films after the protons have propagated through target \#2, being an Al $3 \mu \mathrm{m}$ thick foil, for different proton energies (as indicated above each film), corresponding to different times at which the protons have crossed target (as indicated). Time $\mathrm{t}=0$ corresponds to the time at which the laser B2 irradiates target \#2. Darker signal represents an increase of the proton dose. The $5 \mathrm{~mm}$ scale indicated by the horizontal bar in the image is given in the plane of the RCF. (b)-(h) represent azimuthally averaged profiles of the proton dose modulation recorded in each RCF, normalized to the incident proton dose, i.e., $\delta \mathrm{n} / \mathrm{n}=\left[\mathrm{n}_{\text {recorded }}-\mathrm{n}_{\text {incident }}\right] / \mathrm{n}_{\text {incident }}$ where $\mathrm{n}$ is the proton dose. (i) and (j)-(p) are the same as (a) and (b)-(h) for an Au $3 \mu \mathrm{m}$ thick foil as target \#2. (q) presents the spatial distribution of the protons generated from target \#1, demonstrating that the deflections observed in (a) and (i) are indeed linked with the presence of fields on target \#2. This is the standard distribution of TNSA-generated protons. ${ }^{12}$ In this case target \#1 is a $10 \mu \mathrm{m}$ thick Au foil. (r) displays the spatial distribution of the protons generated from target \#2, i.e., there is no target \#1 in this shot and B1 is blocked. This shows that the proton signal recorded in (a) and (i) is largely dominated by the protons originating from target \#1 since the dose of the protons produced from target \#2 for proton energies $\geq 4.5 \mathrm{MeV}$ is negligible compared with the one of the protons having same energies and produced from target \#1. In this case, target \#2 is a $3 \mu \mathrm{m}$ thick Al foil. The $25 \mathrm{~mm}$ scale shown in between (q) and (r) is given in the plane of the RCF and applies for both (i) and (j). Reproduced with permission from Rev. Sci. Instrum. 86, 043502 (2015). Copyright 2015 AIP Publishing LLC. 

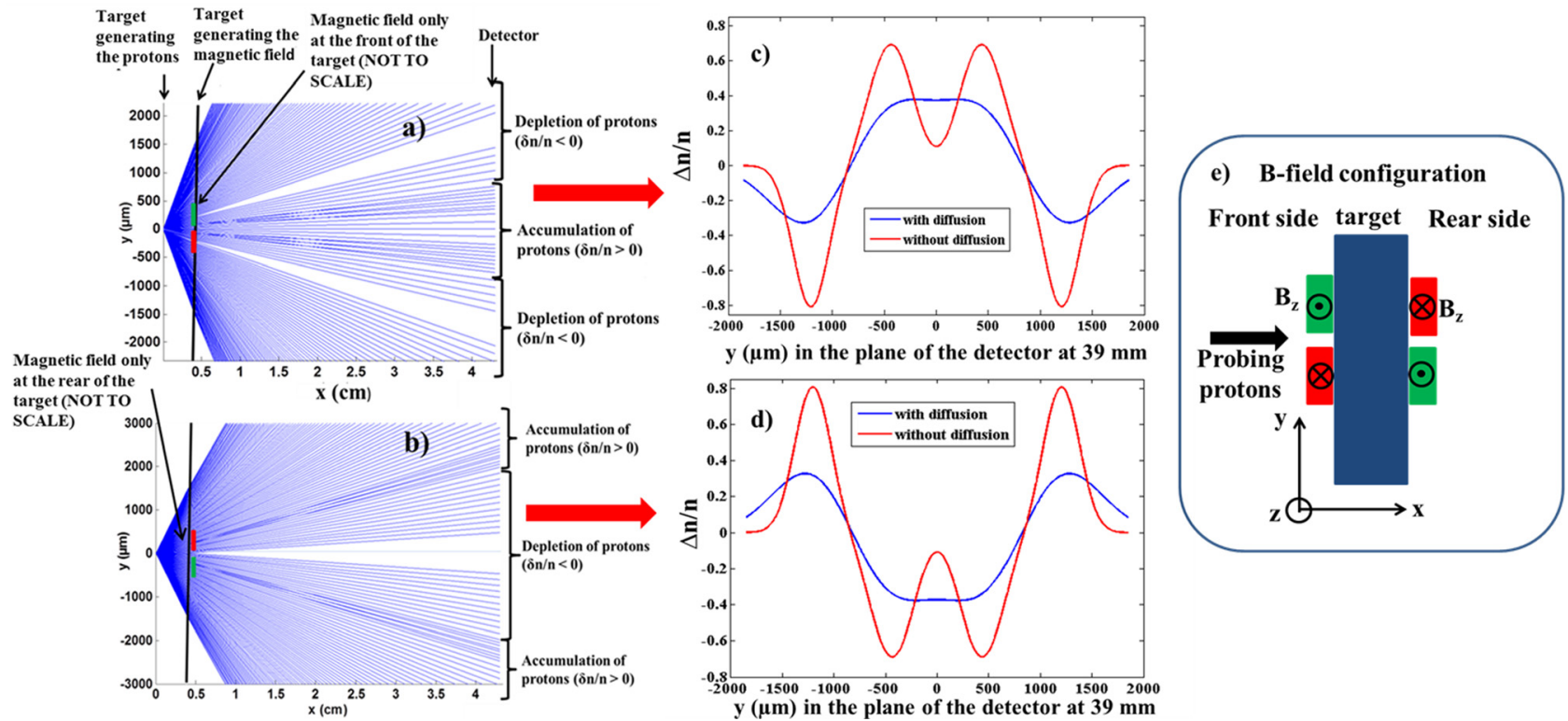

FIG. 3. Particle tracing simulations of protons originating from target \#1 (located at $\mathrm{x}=0$ ) and going through target \#2 (located at $\mathrm{x}=4 \mathrm{~mm}$ ) in two extreme cases: (a) where there are only B-fields at the front of target \#2 without diffusion, and (c) corresponding protons deflections observed onto a detector placed at $39 \mathrm{~mm}$ in the cases with and without diffusion, and (b) where there are only B-fields at the rear of target \#2 without diffusion, and (d) corresponding protons deflections observed onto a detector placed at $39 \mathrm{~mm}$ in the cases with and without diffusion (e) sketch of the B-fields topology used in the simulations.

\section{Topology of the B-fields corresponding to the observations}

In order to understand the B-field topology that produced the deflection patterns observed in Figure 2, we performed particle tracing simulations. As shown in Figure 3(e), the B-fields are modeled as two toroids, one on each surface of target \#2, with the two torus axes parallel and along the target normal. On each target surface, the B-field is oriented clock-wise around the local target normal. ${ }^{1-3}$ The protons, originating from target $\# 1$, and having a $20^{\circ}$ divergence angle at the source $\mathrm{e}^{12,27}$ as in the experiment, are propagated through these B-fields, and the deflections they are subjected to are projected on a plane simulating the RCF. We simulated two extreme cases: one (a) where we consider only the B-fields that are present on the front side of target \#2 (the one facing target \#1 and the probing protons), and the second (b) where we consider only the B-fields that are present on the target rear surface. In both cases, the B-field strength is 8 MG. We do not here consider the B-fields that develop inside the target since, as will be shown later in Section III B 2, they induce negligible deflections upon the protons compared with what the surface B-fields do.

Case (a) results on the detector in a concentration of protons in the center (see Figure 3(a)). On the contrary, case (b) results on the detector in a central depletion and an accumulated outer ring (see Figure 3(b)). The ray-tracing shown in Figures 3(a) and 3(b) do not include the scattering (diffusion) of the probing protons through the target. Figures 3(c) and 3(d) present the influence the scattering has on the final proton modulation as seen on the RCF. In the case of a front surface B-field, the scattering has a tendency to smooth the two central peaks observed in Figure 3(c) in only one central dot (corresponding to increased dose). For a rear surface B-field, similarly, the scattering smoothes the modulation in the dose depletion at the center (see Figure 3(d)). In both cases, the amplitude of the deflection $\Delta \mathrm{n} / \mathrm{n}$ is lower and the dose peaks and depressions are wider.

With these two cases, we can then interpret the results of Figure 2: the early phase where protons are accumulated on an outer ring corresponds to case (b), i.e., to B-fields predominantly on the target rear surface. Note that this situation is similar for both targets since the proton deflections recorded at $\mathrm{t} \sim 0-1$ ps are similar for both targets. Very late times where protons are accumulated in a central dot correspond to the opposite case (a), i.e., to B-fields predominantly on the target front surface. This late-time situation is also similar for both targets (see Figures 2(p) and 2(h)). The intermediate situation, where the outer ring progressively disappears and the inner concentration of protons increases thus corresponds to a transition from the B-fields being predominant on the rear to being predominant on the front. This transition is very short and abrupt in the case of the $\mathrm{Al}$ target (see Figures 2(a)-2(h)) while it is more progressive in the case of the Au target (see Figures 2(i)-2(p)).

More quantitatively, we can deduce, from the particle tracing, the B-fields strength that induces the observed proton deflections. This is shown in detail in Fig. 4 of Ref. 11. In the case of the Al target, the rear surface B-field is observed to vanish at $\sim 15-20 \mathrm{ps}$, with a decrease $\sim \exp (-\mathrm{t}$ [ps]/4), while the front B-field, of the order of $8 \mathrm{MG}$, remains almost constant. In the case of the Au target, since there is significantly more scattering of the probing protons, the fitting of the data to retrieve an unequivocal distribution of the B-fields is more difficult. At this latest time, we can only state that the front $\mathrm{B}$-field is $\sim 6 \mathrm{MG}$ and is predominant (as attested by the strong central peak in the proton dose), while the rear B-field is between 0 and $8 \mathrm{MG}$.

We will now analyze, with the help of models and numerical simulations of the laser-matter interaction, the origin 
of these B-fields and of the transition of their predominant location from the rear to the front of target \#2.

\section{ANALYSIS AND DISCUSSION}

\section{A. Mechanisms of B-fields generation}

There are different possible mechanisms of B-fields self-generation; they are schematically illustrated in Fig. 4. Following Ref. 28, Faraday's law can be expressed as:

$$
\begin{aligned}
\frac{\partial \boldsymbol{B}}{\partial t}= & \underbrace{\nabla \times\left(\left(\boldsymbol{v}+\boldsymbol{V}_{N}\right) \times \boldsymbol{B}\right)}_{\text {First }}-\underbrace{\nabla \times\left(\boldsymbol{J}_{\boldsymbol{R}} \times \frac{\boldsymbol{B}}{n_{e} e}\right)}_{\text {Second }} \\
& +\underbrace{\frac{1}{n_{e} e} \nabla T_{e} \times \nabla n_{e}}_{\text {Third }} \underbrace{-\nabla \times\left(\eta \boldsymbol{J}_{\boldsymbol{R}}\right)}_{\text {Fourth }} .
\end{aligned}
$$

The first term (where $v$ is the fluid velocity and $V_{N}$ is the Nernst velocity ${ }^{29}$ ) represents the B-field convection, the second and the third terms are source terms induced, respectively, by the hot electron forward current $\mathrm{J}_{\mathrm{F}}$, which also triggers a counter-stream return current $\mathrm{J}_{\mathrm{R}}$ with $\mathrm{J}_{\mathrm{F}}=-\mathrm{J}_{\mathrm{R}}{ }^{30}$ (see Fig. 4(a)), and the thermoelectric effect, related to the non-collinearity of the gradients of the electron temperature ( $T_{e}$, the gradient being mostly along radial, i.e., along y) and of the electron density ( $n_{e}$, the gradient being mostly along longitudinal, i.e., along $\mathrm{x}$ ), and which is also known as the Biermann battery effect ${ }^{31}$ (see Fig. 4(b)). These two terms will obviously not take place on the same time scales, the hot electrons being likely damped quickly ${ }^{32}$ while the hydrodynamic target evolution takes place over long times (ns). ${ }^{33}$ The fourth term is driven by the Ohmic fields $E_{R}=\eta \quad J_{R}$ where $\eta$ is the plasma resistivity that dynamically changes during target heating due to the hot-electron flow.

Hot electrons are mainly generated, in our case, through the $J \times B^{34}$ and Brunel $^{35}$ mechanisms, yielding electrons with energy $>600 \mathrm{keV}$. Having MegaAmpere (MA) current, they propagate along the surface ${ }^{36}$ as well as inside the target. ${ }^{6}$ For small incidence angle on target (B2 irradiates target $\# 2$ at $30^{\circ}$, as stated earlier), it has been shown experimentally in various conditions ${ }^{36,37}$ as well as theoretically, ${ }^{38}$ in conditions relevant to our study, that the population of electrons injected in the target is predominant over the electrons confined at the target front. The injection of a large population of electrons inside the target triggers, as shown by Bell et al., ${ }^{39}$ the generation of a comparable cold return electron current $J_{R}$ in order for the forward net current not to exceed the Alfven limit. ${ }^{40}$ Note also that since target \#2 is relatively thin, longitudinal refluxing ${ }^{41}$ quickly takes place in the target. However, since the partial neutralization of the forward electrons by the return current is global and not local, the local non-compensation between the hot and cold return electron current gives rise, through the so-called "fountain effect" ${ }^{3,42}$ (see Fig. 4(a)), to an azimuthal self-generated B-field, with a strength on the order of tens of MG. The B-field induced by the thermoelectric effect can be expressed as: ${ }^{28}$

$$
B[T] \approx 1.0004\left(\frac{\mu \mathrm{m}}{L_{n}}\right)\left(\frac{T_{e}}{\mathrm{eV}}\right)\left(\frac{\mu \mathrm{m}}{L_{T}}\right)\left(\frac{\tau}{\mathrm{ps}}\right),
$$

where $L_{n}$ is the longitudinal (x-wise) density gradient scale length, $T_{e}$ is the bulk electron temperature, $L_{T}$ is the radial (y-wise) temperature gradient scale length, and $\tau$ is the laser pulse duration. As expressed by Eq. (3.2), the field strength is directly dependent on the density and temperature gradients; hence, a poor temporal contrast of the laser, inducing an early plasma expansion and long gradients, will lead to low B-fields. Previous studies of Ref. 15 showed that this Bfield is long-lived (few tens of ps) and of high strength (1-3 MG) at the target front side.

In order to understand what the source of the B-fields observed in the experiment is, as well as how and why the B-fields transit from being predominant at the rear to being predominant at the front, we will use numerical simulations. To model the B-fields induced by the hot electrons, we will first use a kinetic description of the plasma, i.e., particle-incell simulations. Then, to model the thermoelectric B-fields, we will use hydrodynamic-radiative simulations.

\section{B. Numerical simulations of the B-fields induced by the hot electron current}

To assess the growth and evolution of the B-fields induced by the hot electrons (the refluxing of which is intrinsically accounted for in the simulations), we have performed 2D PIC simulations of the interaction between B2 and target \#2 (see Fig. 5) using the PICLS code. ${ }^{43}$ As in the
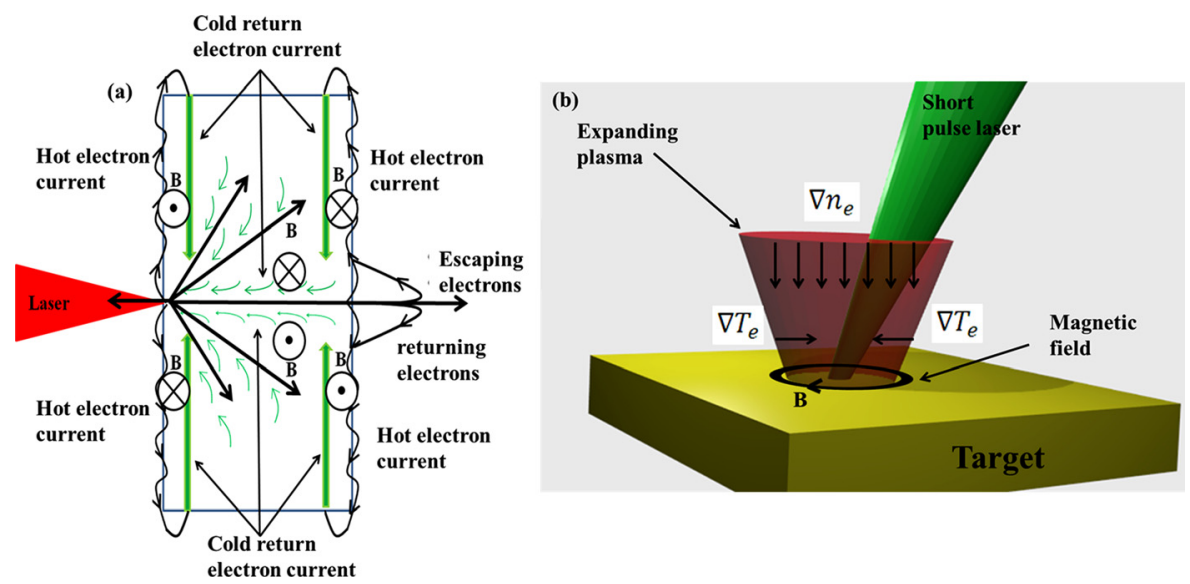

FIG. 4. Schematic representation of the B-field related to (a) the hot electron currents and (b) the non-colinearity of the gradients of the electron temperature ( $T_{e}$, the gradient being mostly along radial, i.e., along y) and of the electron density $\left(n_{e}\right.$, the gradient being mostly along longitudinal, i.e., along $\mathrm{x}$ ). 


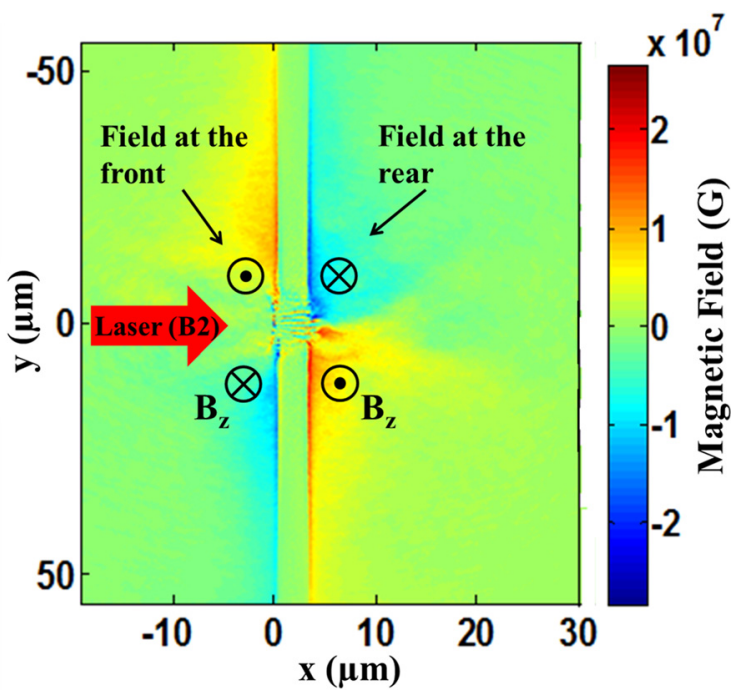

FIG. 5. Map of the self-generated magnetic field on target \#2 (3 $\mu$ m thick $\mathrm{Au}$ ), at $\sim 1 \mathrm{ps}$ after the beginning of the interaction, as simulated using the PICLS code.

experiment, the laser in the simulation is at the fundamental wavelength $\lambda=1.06 \mu \mathrm{m}$. It is incident on the target along the target normal, has a peak intensity of $\mathrm{I}=5 \times 10^{19} \mathrm{~W} \mathrm{~cm}^{-2}$, propagates from the left to the right of the simulation box, and irradiates a $3 \mu \mathrm{m}$ thick solid target at $400 \mathrm{n}_{\mathrm{c}}$ of $\mathrm{Al}$ or $\mathrm{Au}$. The targets include a thin layer $(100 \mathrm{~nm})$ of hydrogen on the front and rear surfaces in order to model the adsorbed impurities on the target surfaces. ${ }^{44}$ Due to the relatively long duration of the laser pulse (700 fs FWHM) and to minimize edge effects, a simulation box with high dimension is necessary; it is here of $100 \mu \mathrm{m} \times 120 \mu \mathrm{m}$. The resolution of the simulation is $80 \mathrm{mesh} / \lambda$, and the total number of particles is $\sim 7.5 \times 10^{6}$. Collisions implemented in the code based on the model of Takizuka and $\mathrm{Abe}^{45}$ are used, as well as ionization of the target material, based on the Thomas Fermi model. The duration of the simulation is approximately 2 ps. Fig. 5 displays a typical B-field map at $\mathrm{t} \sim 1$ ps after the beginning of the laser irradiation for a $3 \mu \mathrm{m}$ thick $\mathrm{Au}(\mathrm{Z}=79)$ foil.

It is important to note several limitations intrinsic to running such simulations. Indeed, PIC simulations have a tendency to underestimate the magnetic field due to the $2 \mathrm{D}$ geometry which restricts the recirculation of hot electrons (which takes place in volume in 3D), as well as the amplification of the magnetic field due to thermal instabilities for example, Ref. 46 which can develop perpendicularly to the heat flow and are more important for high $\mathrm{Z}$ materials. Also, due to the finite dimension of the simulation box, we observe in Fig. 5 that the fields extend up to the edge of the box (where we use periodic boundary conditions). As an example, for a time of $t=0.7425 \mathrm{ps}$ the B-field at the edge of the simulation box is still of $\sim 7 \mathrm{MG}$. Its radial extension is thus obviously limited by the size of the simulation box, which is constrained (in our case) by computing power limitations.

One can distinguish in Fig. 5 two strong, toroidal-shape magnetic fields developing on the target surfaces. We can also distinguish some smaller scale magnetic fields inside the target, which will be detailed later on.

\section{Analysis of the magnetic fields developing on the target surfaces}

The temporal evolution of the maximum of the B-field on each surface of the target as calculated in the simulation is shown in Fig. 6. These values are averaged over a box that is $0.5 \mu \mathrm{m}$ deep along the $\mathrm{x}$ axis and $5 \mu \mathrm{m}$ long along the $\mathrm{y}$ axis (see Fig. 5) around the location of the maximum field strength position. For both targets, we observe that the B-fields grow rapidly over a few 100s of fs and are stronger on the target rear side, which is well consistent with what we deduced in Section IIC from the experimental observations. This asymmetry is more pronounced in the case of the $\mathrm{Au}$ target where the maximum B-field is of the order of 16-20 MG on the front surface while it is 25-28 MG at the rear surface. The asymmetry in the field strength is due to the maximum electron density which is observed in the simulation to be higher at the rear side than at the front side of the target. As mentioned earlier, this predominance of electrons being injected into the target and contributing to the rear B-field over the electron confined at the target front, is consistent with previous experimental ${ }^{36,37}$ and theoretical studies. ${ }^{38}$ It is due to the fact that we use a small incidence angle on target \#2. We have also tested in the simulations the influence of a large incidence angle $\left(70^{\circ}\right)$, keeping otherwise the same parameters for the PICLS simulations, and found then, consistently with those previous works, ${ }^{36,38}$ that the situation becomes reversed, the electrons are predominantly at the target front, and hence the B-field becomes there predominant. This could be used to obtain a reversal effect of the action of the B-field, e.g., to focus protons at early time instead of defocusing them (see Figure 2).
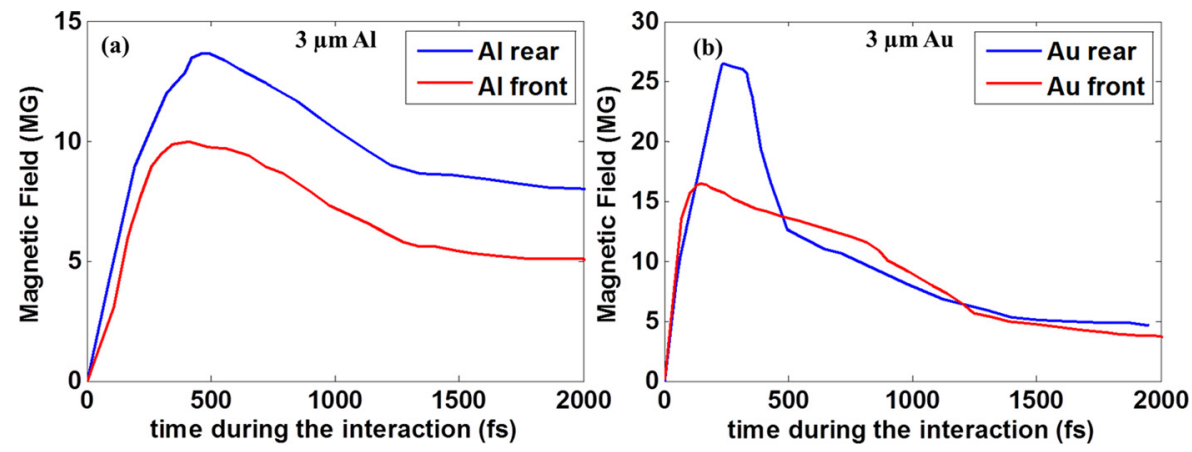

FIG. 6. Temporal evolution of the peak strength of the B-field on both target surfaces as observed in the PICLS simulations for (a) a $3 \mu \mathrm{m}$ thick $\mathrm{Al}$ foil and (b) a $3 \mu \mathrm{m}$ thick Au foil. 

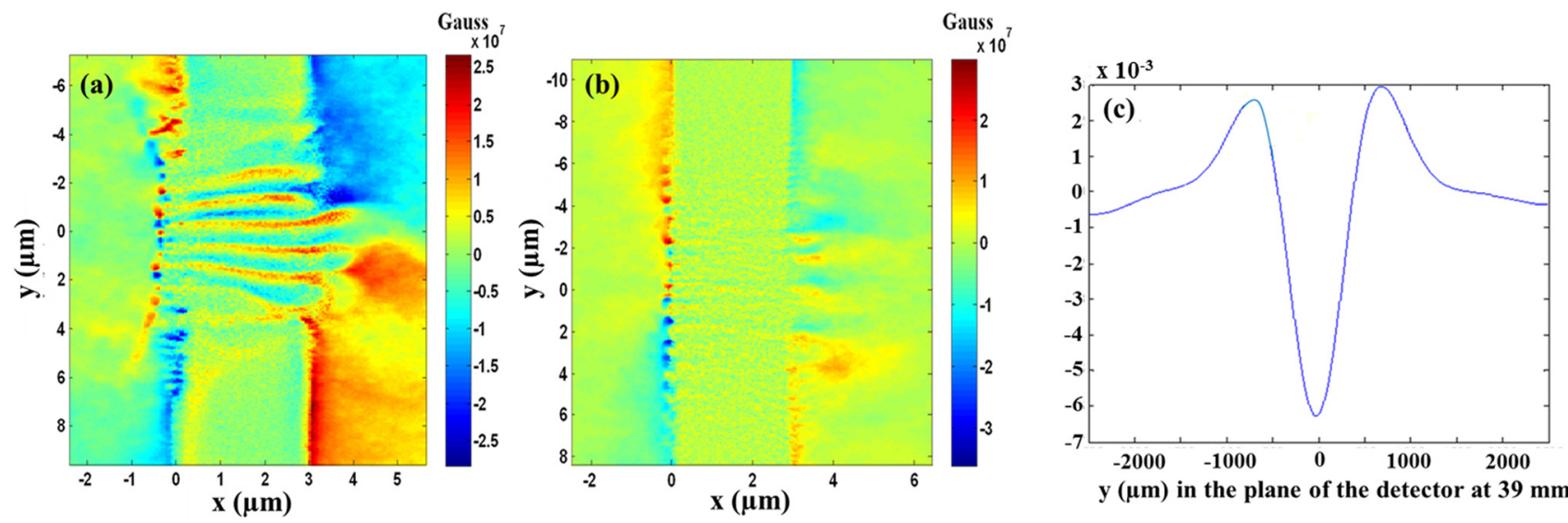

FIG. 7. Self generated magnetic field in the PIC simulations inside the target and averaged over a laser period $\boldsymbol{B}_{Z}$ for (a) the case of the $3 \mu \mathrm{m}$ thick Au and (b) the case of the $\mathrm{Al} 3 \mu \mathrm{m}$ thick target, both at $1 \mathrm{ps}$ after the beginning of the interaction. (c) Proton deflection $(\Delta \mathrm{n} / \mathrm{n})$ observed onto a detector placed at $\mathrm{t}=39 \mathrm{~mm}$ for the map of (a).

\section{Analysis of the self generated magnetic field developing inside the target}

Fig. 7(a) presents the central region of the B-field map shown in Fig. 5, detailing the B-fields developing within the Au target, and Fig. 7(b) shows the same region for the Al target. Both display filamented magnetic structures resulting from the interpenetration of the forward and return electron currents, ${ }^{47}$ although these are significantly stronger in the case of the Au target. The typical magnetic channels for the case of the Au target are of the order of $0.45 \mu \mathrm{m}$ in diameter with an average strength of $10 \mathrm{MG}$.

Inside the target, i.e., at solid density, the principal source of the magnetic field is given by the fourth term of Equation (3.1) that can be developed as

$$
\frac{\partial \boldsymbol{B}}{\partial t} \sim \eta \nabla \times \mathbf{J}_{\mathbf{R}}+\mathbf{J}_{\mathbf{R}} \nabla \times \eta .
$$

We have investigated in detail these B-fields in a previous work $^{6}$ where we showed that due to the small heat capacity and ionization level of $\mathrm{Al}$, there is compensation between the first term and the second term of the right hand side of Equation (3.3). This leads to relatively low B-fields in the case of the $\mathrm{Al}$ target. In opposition, the high heat capacity and ionization level of Au leads to a different evolution of the resistivity inside the target, and the two terms of Equation (3.3) do not compensate each other. In this case, the first term of the right hand side is dominant, allowing the generation of a multi-MG magnetic field (see Fig. 7(a)), albeit in a very filamentary form, likely due to the strong refluxing of the hot electrons at play in such thin target. ${ }^{41}$

\section{Comparison with the experimental data at early times}

To compare quantitatively the PIC simulations with the experiment, we simulate the probing proton deflections through the simulated B-fields by means of a particle tracing code. This code takes into account the divergence of the protons source, as well as the diffusion through the target (see Fig. 3).

First, we tested in a simple case the influence of the parameters of the B-field (radial and longitudinal extent, as well as strength) on the simulation of the dose modulation. From Figure 8, we can observe the following things: (a) the amplitude of the B-field mostly impacts the amplitude of the proton dose modulation $\Delta \mathrm{n} / \mathrm{n}$, and not its radial location on the film (see Figure 8(c)), (b) the radial location on the film of the peak of the proton dose modulation depends mostly on the B-field radial extent (R), and very little on its longitudinal extent (compare Figures 8(b) and 8(d)).

Then, we performed this particle-tracing simulation using the full B-field map obtained from the PIC simulation shown in Figure 5, i.e., including the B-field within the target shown in Figure 7 . In the simulation the protons are
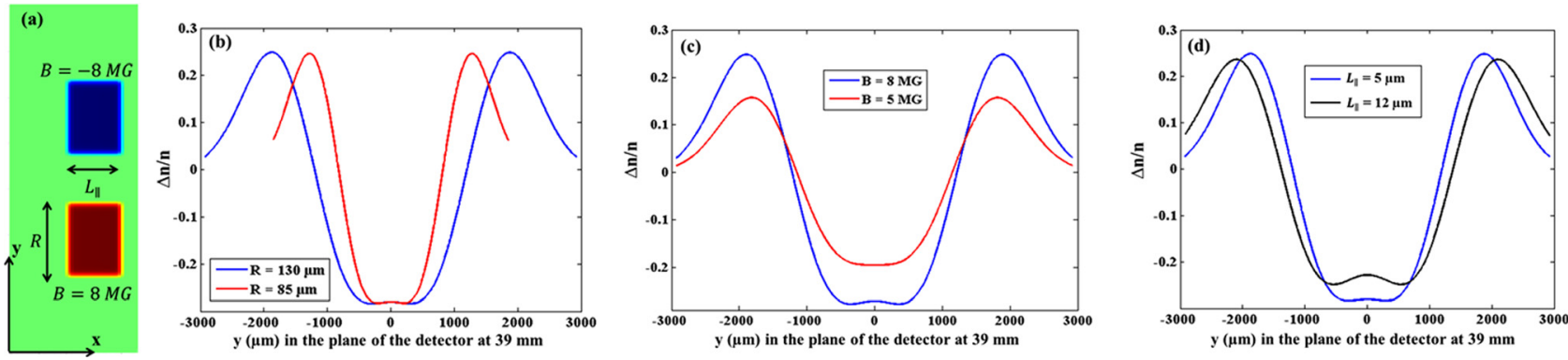

FIG. 8. (a) Arrangement of B-field that are used in the particle tracing simulation, mimicking the B-field at the target rear (see Figure 5). The protons are launched from the left, at the position of the proton source target, from a point source, and after being propagated through the B-field, are collected on the detector. Simulation of the modulation induced on the protons by varying (b) the radial extent of the B-field (R); (c) the B-field strength; and (d) the longitudinal extent of the B-field $\left(\mathrm{L}_{/ /}\right)$. 
propagated along the $\mathrm{x}$-axis, starting from the foil source foil, up to the RCF detector. We note that the deflections induced on the probing protons by the sole B-field within the target (shown in Figure 7) are negligible, as tested by performing particle-tracing simulations solely with those fields (see Fig. 7(c)). The surface B-fields are largely predominantly inducing the observed proton deflections. The result of the simulation is shown in Fig. 9 where it is compared with the earliest time of the experimental proton probing data, the second temporal frame of the experimental results being already at a later time than what can be simulated with the PIC code. Overall, the pattern of the simulated proton deflections is consistent with the experimental ones. Notably, as in the experiment, the simulated proton patterns are similar between the $\mathrm{Au}$ and $\mathrm{Al}$ targets at $\mathrm{t} \sim 1 \mathrm{ps}$. In particular, the amplitude of the proton modulation is well reproduced, which implies that the strength of the simulated Bfields is reflecting the fields present on the target in the experiment. However, we notice that the radial extent of the peak of the proton modulation (the outer ring in the proton deflection maps) is larger in the experiment than in the simulated. This is principally due to limitation of the size of the simulation box in the y (transverse, see Fig. 5) direction, as shown in Figure 8. For the B-fields to extend in the y direction over more than $200 \mu \mathrm{m}$ such that the experimental radial deflection can be reproduced, this would imply a huge simulation box which is not realistic in terms of computing time, owing to the resolution $(80 \mathrm{mesh} / \lambda)$ we need to respect. We should also note one subtle point about the similarity of the proton patterns between $\mathrm{Al}$ and $\mathrm{Au}$ in both experiment and simulation. At first, since the strength during the phase 0-1 ps of the B-field are not the same between $\mathrm{Al}$ and $\mathrm{Au}$ in the
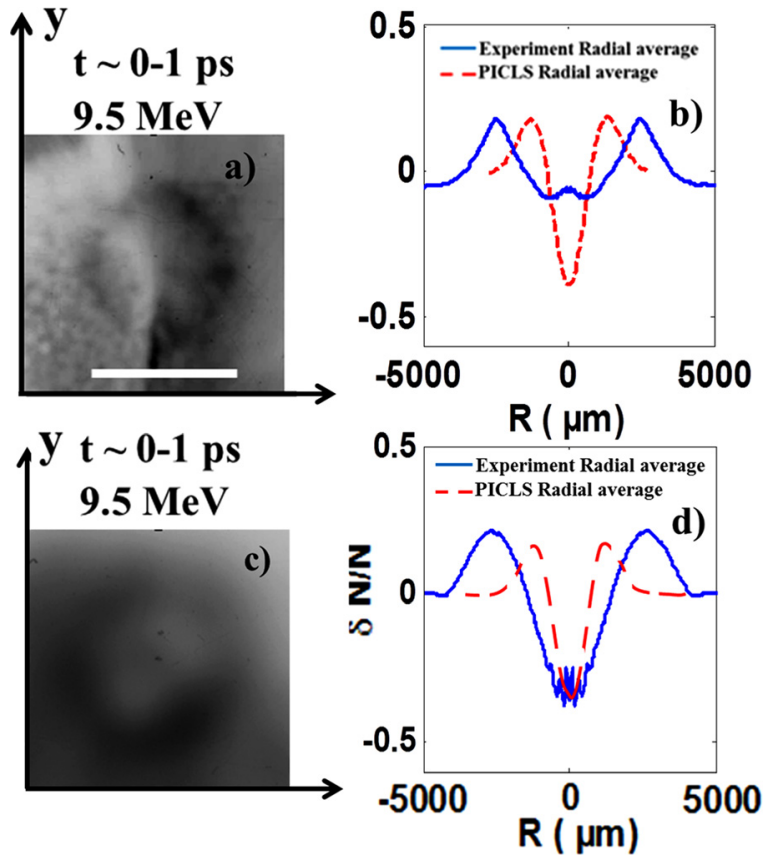

FIG. 9. Experimental results at $t=0-1$ ps after the beginning of the interaction for (a) a $3 \mu \mathrm{m} \mathrm{Al}$ and (b) comparison with PICLS simulation (in dashed red) (at $\sim 1 \mathrm{ps}$ ) of the radial average modulation of dose and for (c) a $3 \mu \mathrm{m}$ $\mathrm{Au}$ and (d) comparison with PICLS simulation (in dashed red) of the radial average modulation of dose. simulation, one could expect this difference to translate into a difference between the simulated patterns. This is however not what is experimentally observed and this is due to diffusion (scattering of the protons) in target \#2 as the probing protons pass through, the diffusion being higher in $\mathrm{Au}$ than in Al. As shown earlier in Fig. 8, the strength of the B-fields plays a role only in the amplitude of the $\Delta n / n$ and in that case, without diffusion, the $\Delta \mathrm{n} / \mathrm{n}$ should be higher in the case of the Au target than in the case of the Al target. However, the diffusion effectively decreases the $\Delta \mathrm{n} / \mathrm{n}$ amplitude (see also the influence of the diffusion in Fig. 3) for the Au target allowing to compensate the effect due to the B-fields strength.

The strength of the B-fields at the end of the simulation (2 ps) for both the $\mathrm{Al}$ and $\mathrm{Au}$ targets remains extremely high (few MG). The simulations are difficult to pursue over longer times due to computing limitations, but we expect the Bfield to eventually decrease due to damping of the hot electron current by energy transfer to the colder electrons and ions that constitute the bulk of the target. This damping will be investigated in Sec. III C.

\section{Simulation of the transition time from B-fields driven by hot electrons to B-fields driven by the plasma expansion}

The damping of the hot electrons is evaluated using a 1D three-temperature code $\mathrm{e}^{32}$ that calculates the temperature of the hot electrons $T_{h}$, of the cold (bulk) electrons $T_{\text {cold }}$, and of the bulk ions $T_{i}$, and takes into account the expansion of the target. The hot electrons, which are assumed to have a Maxwellian energy distribution, lose energy over time due to (i) collisions with cold electrons and (ii) the development of the resistive electric field within the target that is at the source of the return current. The equation governing the temporal evolution of $T_{h}$, is written as ${ }^{32}$

$$
\begin{aligned}
n_{h} k_{B} \frac{d T_{h}}{d t} & =-n_{h} S_{h c}-\eta\left(T_{i}, T_{\text {cold }}\right) J_{R}^{2} \\
& =-n_{h}\left|\frac{d E_{h}}{d t}\right| v_{h}-\eta\left(T_{i}, T_{\text {cold }}\right) J_{R}^{2},
\end{aligned}
$$

with $n_{h}$ being the hot electron density, $T_{h}$ the hot electron temperature, $E_{h}$ the hot electron energy, $\eta\left(T_{i}, T_{\text {cold }}\right)$ the resistivity depending on the cold electron temperature and of the ion temperature, $J_{R}$ the return current on the cold electrons (as earlier), and $S_{h c}$ the stopping power due to free and bound electrons and plasmons. The hot electron density $n_{h}$ can be estimated using an energy balance equation between the absorbed fraction $(f)$ of the laser power and the power that is developed by the hot electrons: $f I_{l}=n_{h} v_{h} T_{h}$. Using $\mathrm{f} \sim 0.1,{ }^{48}$ for a laser intensity $I_{l}=1 \times 10^{19} \mathrm{~W} \mathrm{~cm}{ }^{-2}$, which leads to $T_{h} \sim 0.6 \mathrm{MeV}$ according to the scaling of Ref. 49, we obtain $n_{h} \sim 3.8 \times 10^{20} \mathrm{~cm}^{-3}$, and for $I_{l}=5 \times 10^{19} \mathrm{~W} \mathrm{~cm} \mathrm{~cm}^{-2}$, and $T_{h} \sim 0.823 \mathrm{MeV}$, we obtain $n_{h} \sim 1.4 \times 10^{21} \mathrm{~cm}^{-3}$.

Figure 10 illustrates the temporal evolution of $T_{h}$ in the $\mathrm{Al}$ and Au targets. In Figure 10(a), we observe that for the Al target the thermalization of the hot electrons (i.e., when $T_{h}=T_{c}=T_{i}$ ) takes place around $29 \mathrm{ps}$ for $I_{l}=5 \times 10^{19} \mathrm{~W} \mathrm{~cm}^{-2}$; the same calculation done for $I_{l}=1 \times 10^{19} \mathrm{~W} \mathrm{~cm}^{-2}$ yields a damping 

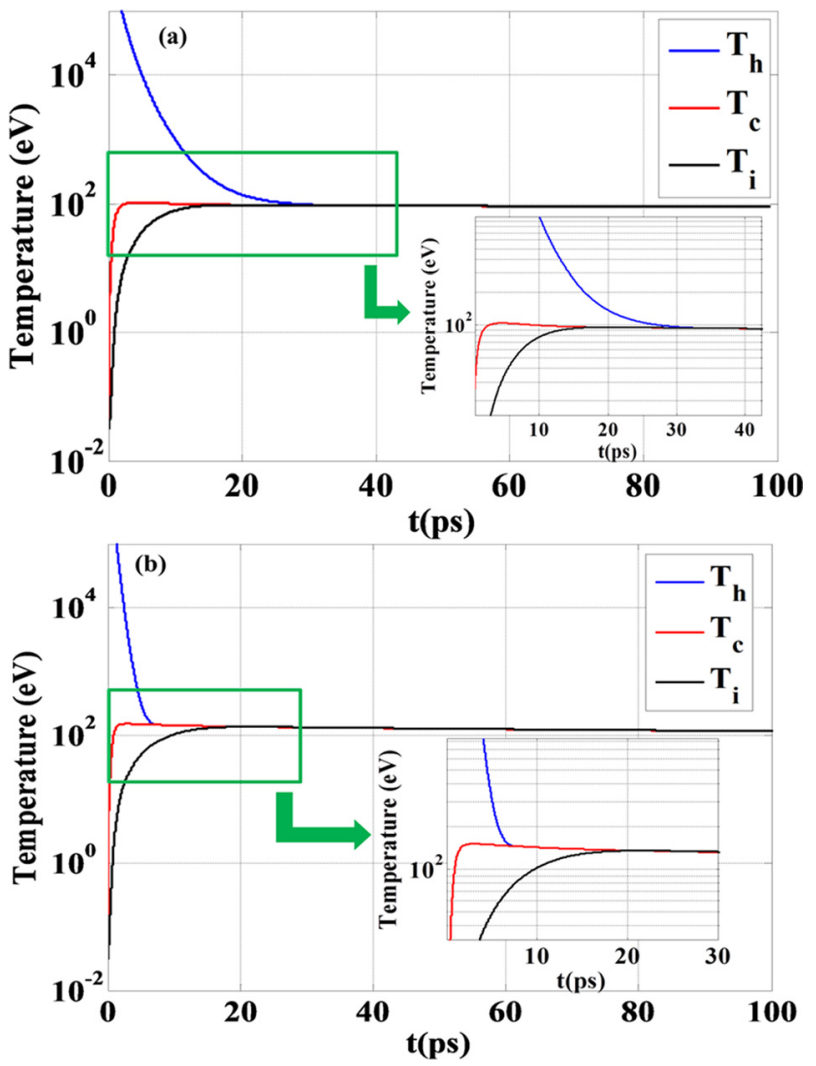

FIG. 10. Temporal evolution of $T_{h}$ (the hot electron temperature), $T_{\text {cold }}$ (the cold electron temperature), and $T_{i}$ (the ion temperature) within (a) a $3 \mu \mathrm{m}$ thick Al target and (b) a $3 \mu \mathrm{m}$ thick Au target, in the case of an irradiation by a laser having an intensity of $5 \times 10^{19} \mathrm{~W} \mathrm{~cm}^{-2}$ and a pulse duration of $700 \mathrm{fs}$.

time of $14 \mathrm{ps}$. Such rapid decay of $T_{h}$ is related to the fast ionization and heating of the $\mathrm{Al}$ target. In the case of the Au target, the damping of the hot electrons also takes place $\sim 10-20 \mathrm{ps}$, as shown in Figure 10(b).

These hot-electron damping times are consistent with the observations in Figure 2 since for both targets we can observe that after 10-20 ps the strong outer ring of proton accumulation, which is associated with strong rear B-fields driven by hot electrons, vanishes. After the hot electrons are damped, the remaining source of B-fields is the thermoelectric effect which is analyzed in Sec. III D.

\section{Simulations of the B-fields driven by plasma hydrodynamic expansion into vacuum}

The late-time thermoelectric B-fields can be assessed in two ways: analytically and numerically. Let us first analytically assess the strength of the front-side and rear-side B-fields that are self-generated by target hydrodynamic expansion. For this, we can rely on Eq. (3.2) which requires knowledge of the longitudinal density $\left(L_{n}\right)$ and radial temperature gradients $\left(L_{T}\right)$. To assess $\left(L_{n}\right)$, in a 1D model of isothermal plasma expansion, we can simply write ${ }^{50}$

$$
L_{n}[\dot{A}]=c_{s} t \sim 3\left(\frac{T_{e}}{\mathrm{keV}}\right)^{\frac{1}{2}}\left(\frac{Z^{*}}{A}\right)^{\frac{1}{2}} \tau_{\mathrm{fs}},
$$

where $T_{e}$ is the electron temperature, $Z^{*}$ is the effective ion charge, $A$ is the atomic number, and $\tau_{\mathrm{fs}}$ is the laser pulse duration in femtosecond. For the $\mathrm{Al}$ target $(A=13)$, we have evaluated in Sec. III C that $T_{e} \sim 80-100 \mathrm{eV}$ (the equilibration temperature, see Figure 10). We also have: $\tau_{\mathrm{fs}}=700 \mathrm{fs}$ and $Z^{*}=9$. Hence, one obtains $L_{n} \sim 0.05-0.1 \mu \mathrm{m}$.

To assess the B-field at the target front, where there has been a strong local energy deposition by the laser, we can use for the radial temperature gradient the value given by the PIC simulation at the longest simulated time, i.e., $L_{T} \sim 2 \mu \mathrm{m}$. Combined with $L_{n}$, this yields $\mathrm{B}_{\text {front }} \sim 8 \mathrm{MG}$, which compares quite well with the strength deduced from our data (see Section II C). Here we can see the major interest of having used a plasma mirror for the laser beam B2 in order to prevent the generation of a pre-plasma where the energy deposition would have been diluted, which would have led to a low B-field strength at the target front.

To assess similarly the B-field at the target rear, we have to consider that the heating is much more radially distributed since it is induced by the fast electrons that are spread widely from the target front over a large radial area at the target rear. ${ }^{13}$ Here, we can use the results of Ref. 22 to retrieve the rearsurface radial temperature gradient. Indeed, Ref. 22 presents measurements of the rear-surface radial distribution of density and temperature for $\mathrm{Al}$ targets of various thicknesses laserirradiated at an intensity of $1-5 \times 10^{19} \mathrm{~W} \mathrm{~cm}^{-2}$. Such laser intensity is similar as the one used in the present experiment; hence, the average fast electron energy and angular spread in the target, i.e., the rear surface electron sheath radial extent, are similar. There, $L_{T}$ was measured to be $\sim 30-50 \mu \mathrm{m}$. Combined with $L_{n}$, this yields (still using Eq. (3.2)), $\mathrm{B}_{\text {rear }} \sim 50 \mathrm{kG}$. The strong asymmetry between the front and rear surface magnetic fields thus deduced at late times is again quite consistent with what was deduced in Section II C from the experimental data.

To complement these analytical estimates, we simulated the target hydrodynamic expansion using the 2D MHD code CHIC. ${ }^{51}$ The input parameters are the following: for the plasma on the front surface of the target, we used the density and temperature spatial distributions inferred from PICLS at long time (1.65 ps), while for the rear surface, we adopted the density and temperature distributions that were experimentally measured. ${ }^{22}$ Figure 11 shows the result of the simulation for a $3 \mu \mathrm{m}$ thick $\mathrm{Al}$ target. A strong asymmetry is observed between the front and the rear surface of the target, consistently with the above-given analytical estimates. The simulated B-field strength on the front surface is $\sim 4$ times higher than the one on the rear surface of the target and it increases with time. However, we note that the simulated field strength disagrees compared with what we can deduce from the experimental proton deflection maps. This is probably related to the fact that we inject into the MHD code, for the front side, the values obtained from the PIC code at still early time, i.e., at a time where the hot electrons have not relaxed, which leads to underestimating the bulk temperature and overestimating the temperature scale-length, and hence underestimating the B-field (see Equation (3.2)).

\section{CONCLUSION}

In conclusion, we have investigated experimentally and numerically the B-fields that are self-generated during and 

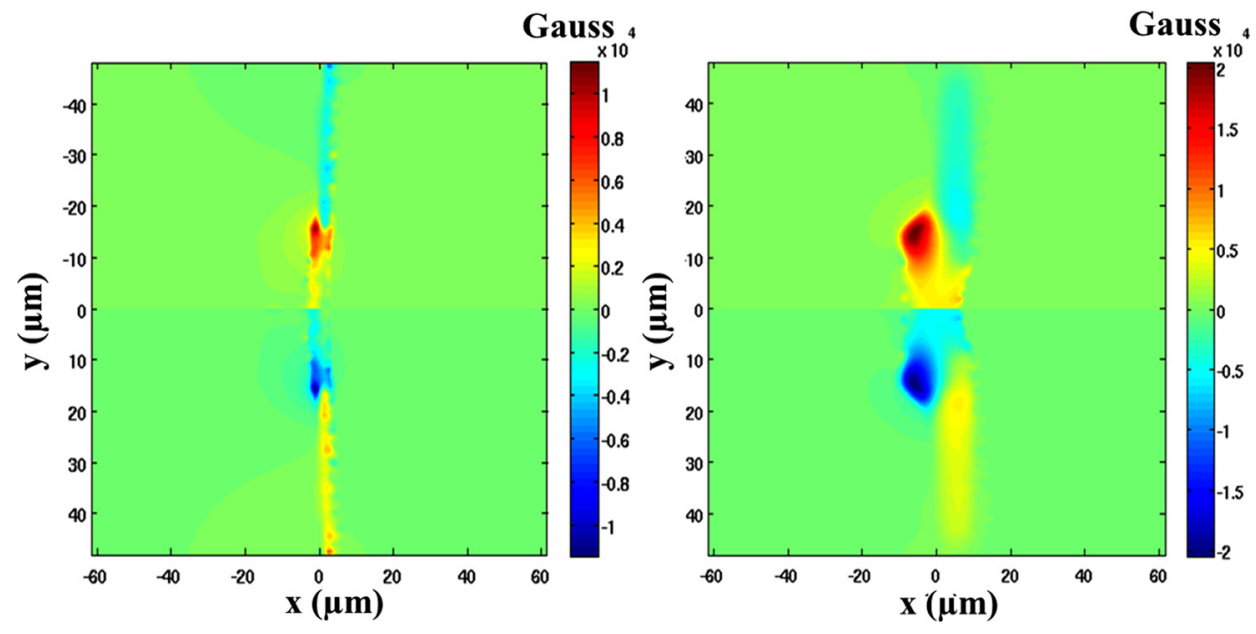

FIG. 11. B-field map simulated by the MHD CHIC code for a $3 \mu \mathrm{m}$ thick $\mathrm{Al}$ target, using input parameters as described in the text, at (a) $10 \mathrm{ps}$ and (b) $40 \mathrm{ps}$. following the interaction of a high contrast, high intensity laser pulse with thin targets of $\mathrm{Al}$ and $\mathrm{Au}$. The general topology of the fields that can be deduced from the experimental proton deflection maps is in good agreement with the simulations that can be made at early times (using PIC simulations) as well as at late times (using hydrodynamic simulations). The main result of this investigation is that over long time scales (10s of ps), a strong asymmetry appears between the B-fields present at the front and the rear surfaces of the target, with the latter vanishing while the former stays of high strength (8-10 MG). This, as shown by us earlier, ${ }^{11}$ is in particular of interest for focusing charged particles. We also note that the observed fast decrease of the rear B-field in the case of the Al target is likely related to an increased resistivity induced by the strong surface B-field, an effect that needs to be taken into account to correctly evaluate the material resistivity. ${ }^{52}$

\section{ACKNOWLEDGMENTS}

We acknowledge the support of the staff at the Jupiter Laser Facility at Lawrence Livermore National Laboratory. The use of the Jupiter Laser Facility was supported by the U.S. Department of Energy, Lawrence Livermore National Laboratory, under Contract No. DE-AC52-07NA27344. The research leading to these results has received funding from Laserlab-Europe (Grant Agreement No. 284464, EC's seventh framework program) and Grant No. 001528. This work was partly done within the LABEX Plas@Par project and supported by Grant No. 11-IDEX-0004-02 from Agence Nationale de la Recherche. This work was also partly supported by the DFG GRK 1203, by NSERC Grant No. 435416 - Computecanada and SFB/TR18 programs and by EPSRC Grant Nos. EP/K022415/1 and EP/J002550/1. This work was supported in part by the Ministry of Education and Science of the Russian Federation under Contract No. 14.Z50.31.0007.

\footnotetext{
${ }^{1}$ A. R. Bell, F. N. Beg, Z. Chang, A. E. Dangor, C. N. Danson, C. B. Edwards, A. P. Fews, M. H. R. Hutchinson, S. Luan, P. Lee, P. A. Norreys, R. A. Smith, P. F. Taday, and F. Zhou, Phys. Rev. E 48, 2087 (1993).

${ }^{2}$ M. J. Mason and M. Tabak, Phys. Rev. Lett. 80, 524 (1998).

${ }^{3}$ A. Pukhov, Phys. Rev. Lett. 86, 3562 (2001).
}

${ }^{4}$ M. Storm, A. A. Solodov, J. F. Myatt, D. D. Meyerhofer, C. Stoeckl, C. Mileham, R. Betti, P. M. Nilson, T. C. Sangster, W. Theobald, and C. Guo, Phys. Rev. Lett. 102, 235004 (2009).

${ }^{5}$ B. Ramakrishna, S. Kar, A. P. L. Robinson, D. J. Adams, K. Markey, M. N. Quinn, X. H. Yuan, P. McKenna, K. L. Lancaster, J. S. Green, R. H. H. Scott, P. A. Norreys, J. Schreiber, and M. Zepf, Phys. Rev. Lett. 105, 135001 (2010).

${ }^{6}$ Y. Sentoku, E. d'Humières, L. Romagnani, P. Audebert, and J. Fuchs, Phys. Rev. Lett. 107, 135005 (2011).

${ }^{7}$ A. P. L. Robinson, M. H. Key, and M. Tabak, Phys. Rev. Lett. 108, 125004 (2012)

${ }^{8}$ X. H. Yang, M. Borghesi, and A. P. L. Robinson, Phys. Plasmas 19, 062702 (2012)

${ }^{9}$ D. A. MacLellan, D. C. Carroll, R. J. Gray, A. P. L. Robinson, M. P. Desjarlais, D. Neely, and P. McKenna, Plasma Phys. Controlled Fusion 56, 084002 (2014).

${ }^{10}$ H. B. Zhuo, Z. L. Chen, Z. M. Sheng, M. Chen, T. Yabuuchi, M. Tampo, M. Y. Yu, X.H. Yang, C.T. Zhou, K.A. Tanaka, J. Zhang, and R. Kodama, Phys. Rev. Lett. 112, 215003 (2014).

${ }^{11}$ B. Albertazzi, E. d'Humières, L. Lancia, V. Dervieux, P. Antici, J. Böcker, J. Bonlie, J. Breil, B. Cauble, S. N. Chen, J. L. Feugeas, M. Nakatsutsumi, P. Nicolaï, L. Romagnani, R. Shepherd, Y. Sentoku, M. Swantusch, V. T. Tikhonchuk, M. Borghesi, O. Willi, H. Pépin, and J. Fuchs, Rev. Sci. Instrum. 86, 043502 (2015).

${ }^{12}$ P. R. Bolton, M. Borghesi, C. Brenner, D. C. Carroll, C. De Martinis, F. Fiorini, A. Flacco, V. Floquet, J. Fuchs, P. Gallegos, D. Giove, J. S. Green, S. Green, B. Jones, D. Kirby, P. McKenna, D. Neely, F. Nuesslin, R. Prasad, S. Reinhardt, M. Roth, U. Schramm, G. G. Scott, S. TerAvetisyan, M. Tolley, G. Turchetti, and J. J. Wilkens, Phys. Med. 30, 255-270 (2014).

${ }^{13}$ J. Fuchs, P. Audebert, M. Borghesi, H. Pépin, and O. Willi, C. R. Phys. 10, 176 (2009); H. Daido, M. Nishiuchi, and A. S. Pirozhkov, Rep. Prog. Phys. 75, 056401 (2012); A. Macchi, M. Borghesi, and M. Passoni, Rev. Mod. Phys. 85, 751-793 (2013).

${ }^{14}$ M. Tatarakis, I. Watts, F. N. Beg, E. L. Clark, A. E. Dangor, A. Gopal, M. G. Haines, P. A. Norreys, U. Wagner, M.-S. Wei, M. Zepf, and K. Krushelnick, Nature 415, 280 (2002).

${ }^{15}$ M. Borghesi, A. J. MacKinnon, A. R. Bell, R. Gaillard, and O. Willi, Phys. Rev. Lett. 81, 112 (1998).

${ }^{16}$ A. S. Sandhu, A. K. Dharmadhikari, P. P. Rajeev, G. R. Kumar, S. Sengupta, A. Das, and P. K. Kaw, Phys. Rev. Lett. 89, 225002 (2002).

${ }^{17}$ W. Schumaker, N. Nakanii, C. McGuffey, C. Zulick, V. Chyvkov, F. Dollar, H. Habara, G. Kalintchenko, A. Maksimchuk, K. A. Tanaka, A. G. R. Thomas, V. Yanovsky, and K. Krushelnick, Phys. Rev. Lett. 110, 015003 (2013)

${ }^{18}$ L. Romagnani, J. Fuchs, M. Borghesi, P. Antici, P. Audebert, F Ceccherini, T. Cowan, T. Grismayer, S. Kar, A. Macchi, P. Mora, G. Pretzler, A. Schiavi, T. Toncian, and O. Willi, Phys. Rev. Lett. 95, 195001 (2005).

${ }^{19}$ J. R. Rygg, F. H. Séguin, C. K. Li, J. A. Frenje, M. J.-E. Manuel, R. D. Petrasso, R. Betti, J. A. Delettrez, O. V. Gotchev, J. P. Knauer, D. D. Meyerhofer, F. J. Marshall, C. Stoeckl, and W. Theobald, Science 319, 1223 (2008). 
${ }^{20}$ C. A. Cecchetti, M. Borghesi, J. Fuchs, G. Schurtz, S. Kar, A. Macchi, L. Romagnani, P. A. Wilson, P. Antici, R. Jung, J. Osterholtz, C. A. Pipahl, O. Willi, A. Schiavi, M. Notley, and D. Neely, Phys. Plasmas 16, 043102 (2009).

${ }^{21}$ G. Sarri, A. Macchi, C. A. Cecchetti, S. Kar, T. V. Liseykina, X. H. Yang, M. E. Dieckmann, J. Fuchs, M. Galimberti, L. A. Gizzi, R. Jung, I. Kourakis, J. Osterholz, F. Pegoraro, A. P. L. Robinson, L. Romagnani, O. Willi, and M. Borghesi, Phys. Rev. Lett. 109, 205002 (2012).

${ }^{22}$ P. Antici, J. Fuchs, M. Borghesi, L. Gremillet, T. Grismayer, Y. Sentoku, E. d'Humières, C. A. Cecchetti, A. Mančić, A. C. Pipahl, T. Toncian, O. Willi, P. Mora, and P. Audebert, Phys. Rev. Lett. 101, 105004 (2008).

${ }^{23}$ J. A. Stamper, K. Papadopoulos, R. Sudan, S. Dean, E. McLean, and J. Dawson, Phys. Rev. Lett. 26, 1012 (1971).

${ }^{24}$ H. Kapteyn, M. Murnane, A. Szoke, and R. W. Falcone, Opt. Lett. 16, 490 (1991); G. Doumy, F. Quéré, O. Gobert, M. Perdrix, Ph. Martin, P. Audebert, J. C. Gauthier, J.-P. Geindre, and T. Wittmann, Phys. Rev. E 69, 026402 (2004).

${ }^{25}$ J. F. Ziegler, J. P. Biersack, and U. Littmark, The Stopping and Range of Ions in Matter (Pergamon, New York, 1985).

${ }^{26}$ V. L. Highland, Nucl. Instrum. Methods 129, 497 (1975).

${ }^{27}$ A. Mancic, J. Robiche, P. Antici, P. Audebert, C. Blancard, P. Combis, F. Dorchies, G. Faussurier, S. Fourmaux, M. Harmand, R. Kodama, L. Lancia, S. Mazevet, M. Nakatsutsumi, O. Peyrusse, P. Renaudin, R. Shepherd, and J. Fuchs, High Energy Density Phys. 6, 21 (2010).

${ }^{28}$ M. G. Haines, Phys. Rev. Lett. 78, 254 (1997).

${ }^{29}$ A. Nishiguchi, T. Yabe, M. G. Haines, M. Psimopoulos, and H. Takewaki, Phys. Rev. Lett. 53, 262 (1984).

${ }^{30}$ P. Kolodner and E. Yablonovitch, Phys. Rev. Lett. 37, 1754 (1976).

${ }^{31}$ S. I. Braginskii, Reviews of Plasma Physics, edited by M. A. Leontovich (Consultants Bureau, New York, 1965), Vol. I.

${ }^{32}$ P. Antici, L. Gremillet, T. Grismayer, P. Mora, P. Audebert, M. Borghesi, C. A. Cecchetti, A. Mancic, and J. Fuchs, Phys. Plasmas 20, 123116 (2013).

${ }^{33}$ L. Lancia, B. Albertazzi, C. Boniface, A. Grisollet, R. Riquier, F. Chaland, K.-C. Le Thanh, Ph. Mellor, P. Antici, S. Buffechoux, S. N. Chen, D. Doria, M. Nakatsutsumi, C. Peth, M. Swantusch, M. Stardubtsev, L. Palumbo, M. Borghesi, O. Willi, H. Pépin, and J. Fuchs, Phys. Rev. Lett. 113, 235001 (2014).
${ }^{34}$ W. L. Kruer, Comments Plasma Phys. Controlled Fusion 9, 63 (1985).

${ }^{35}$ F. Brunel, Phys. Rev. Lett. 59, 52 (1987).

${ }^{36}$ Y. T. Li, X. H. Yuan, M. H. Xu, Z. Y. Zheng, Z. M. Sheng, M. Chen, Y. Y. Ma, W. X. Liang, Q. Z. Yu, Y. Zhang, F. Liu, Z. H. Wang, Z. Y. Wei, W. Zhao, Z. Jin, and J. Zhang, Phys. Rev. Lett. 96, 165003 (2006).

${ }^{37}$ P. McKenna, D. C. Carroll, R. J. Clarke, R. G. Evans, K. W. D. Ledingham, F. Lindau, O. Lundh, T. McCanny, D. Neely, A. P. L. Robinson, L. Robson, P. T. Simpson, C.-G. Wahlström, and M. Zepf, Phys. Rev. Lett. 98, 145001 (2007).

${ }^{38}$ T. Nakamura, S. Kato, H. Nagamoto, and K. Mima, Phys. Rev. Lett. 93, 265002 (2004).

${ }^{39}$ A. R. Bell, J. R. Davies, S. Guerin, and H. Ruhl, Plasma Phys. Controlled Fusion 39, 653 (1997).

${ }^{40}$ H. Alfven, Phys. Rev. 55, 425 (1939).

${ }^{41}$ M. N. Quinn, X. H. Yuan, X. X. Lin, D. C. Carroll, O. Tresca, R. J. Gray, M. Coury, C. Li, Y. T. Li, C. M. Brenner, A. P. L. Robinson, D. Neely, B. Zielbauer, B. Aurand, J. Fils, T. Kuehl, and P. McKenna, Plasma Phys. Controlled Fusion 53, 025007 (2011).

${ }^{42}$ P. Koldner and E. Yablonovitch, Phys. Rev. Lett. 43, 1402 (1979).

${ }^{43}$ Y. Sentoku and A. Kemp, J. Comput. Phys. 227, 6846 (2008).

${ }^{44}$ M. Allen, P. K. Patel, A. Mackinnon, D. Price, S. Wilks, and E. Morse, Phys. Rev. Lett. 93, 265004 (2004).

${ }^{45}$ T. Takizuka and H. Abe, J. Comput. Phys. 25, 205 (1977).

${ }^{46}$ D. A. Tidman and R. A. Shanny, Phys. Fluids 17, 1207 (1974).

${ }^{47}$ F. Califano, F. Pegoraro, and S. V. Bulanov, Phys. Rev. E 56, 963 (1997).

${ }^{48}$ J. Fuchs, P. Antici, E. d'Humières, E. Lefebvre, M. Borghesi, E. Brambrink, C. A. Cecchetti, M. Kaluza, V. Malka, M. Manclossi, S. Meyroneinc, P. Mora, J. Schreiber, T. Toncian, H. Pépin, and P. Audebert, Nat. Phys. 2, 48-54 (2006).

${ }^{49}$ M. G. Haines, M. S. Wei, F. N. Beg, and R. B. Stephens, Phys. Rev. Lett. 102, 045008 (2009).

${ }^{50} \mathrm{P}$. Gibbon, Short Pulse Laser Interactions with Matter (World Scientific, 2005).

${ }^{51} \mathrm{Ph}$. Maire, R. Abgrall, J. Breil, and J. Ovadia, SIAM J. Sci. Comput. 29, 1781 (2007); J. Breil and Ph. Maire, J. Comput. Phys. 224, 785 (2007).

${ }^{52}$ S. Kahaly, S. Mondal, G. Ravindra Kumar, S. Sengupta, A. Das, and P. K. Kaw, Phys. Plasmas 16, 043114 (2009). 